protein immune response in the pathogenesis of autoimmune disease and in particular of BD.

\section{FRI0162 CLINICAL FEATURES OF A SET OF PATIENTS WITH ANTI- NEUTROPHIL CYTOPLASMIC ANTIBODIES}

EG Tomero Muriel, G García-Melcón, R Velayos, AM Ortiz, I González-Alvaro. Rheumatology, Hospital de La Princesa, Madrid, Spain

\subsection{6/annrheumdis-2001.222}

\section{Background}

Objectives To analyse characteristics and clinical features of patients with positive antineutrophil cytoplasmic antibodies (ANCA).

Methods Retrospectively we reviewed 71 patients tested for the presence of ANCA using indirect inmunofluorescence (IFF; ptterns pANCA and cANCA) and/or antimieloperoxidase ELISA (aMPO) from 1993 to 1999. The variables observed were age, sex, origin department, clinical manifestations, final diagnosis, disease evolution and treatment.

Results Mean age was 60.2 years (16-97) being women $63.3 \%$. cANCA was positively tested in $42 \%$ of patients and pANCA in 58\%(59\% aMPO). The distribution among departments was: Internal Medicine 22.5\%, Rheumatology 22.5\%, Nephrology 16.5\%, Pneumology 14.9\%, Gastroenterology $13.5 \%$ and Dermatology 12\%. 28 patients suffered from lung affections, 27 had kidney affections (30\% rapidly progressing glomerulonephritis), 18 skin involvement, 19 musculoesqueletal manifestations, 5 hearts affections, 3 neuropathies and 2 eye involvement. Diagnosis were: 5 Wegeners Granulomatosis, 5 Microscopic polyangiitis, 8 pANCA associated vasculities, 4 leucocytoclastic vasculitis, 7 rheumatoid arthritis, 7 inflammatory bowel diseases, 6 systemic inmunolopathologycal diseases, 21 not autoimmune disorders and 8 without final diagnosis. Progress: 42\% suffered from periodical relapses, 28\% without clinical manifestations, 21\% died. $53 \%$ of death were directly caused by ANCA associated vasculitis, $26 \%$ were due to concomitant infections and the rest were caused by non related complications.

Conclusion 1. Despitethe high percentage of detected cANCA by IIF only Wegeners granulomatosis were diagnosed, so the use of ELISA antiproteinase-3 must be used. 2. It was observed a high mortality rate in patients in patients with positive ANCA.

\section{FRI0163 INITIAL ASSESSMENT OF WEGENER'S GRANULOMATOSIS - AN AUDIT OF RHEUMATOLOGICAL PRACTICE}

JS McLaren, RA Luqmani. Rheumatic Diseases Unit, Western General Hospital, Edinburgh, UK

\subsection{6/annrheumdis-2001.223}

Background Accurate initial assessment of organ involvement in Wegener's granulomatosis (WG) is essential to determine immediate and future management. Renal and pulmonary involvement are associated with a worse outcome.

Objectives We audited the initial assessment of patients referred to the Rheumatic Diseases Unit (RDU) who were subsequently confirmed to have WG. As there are no published gold standards on what should be assessed, the following essential items were chosen following consensus discussion: blood pressure (BP), urinalysis (and microscopy if haematuria present) and serum creatinine for renal involvement and chest radiograph (CXR) for pulmonary involvement. Tissue biopsy from symptomatic sites was important to establish evidence of granulomata or inflammation (ear, nose and throat (ENT)), vasculitis (cutaneous/ENT) or focal segmental glomerulonephritis (renal).

Methods All patients with WG referred to the RDU over 8 years until Sept 2000 were identified by search of the computer database. Patients with incomplete medical records and re-referrals were excluded. 35 patients were identified and their medical records and CXRs were reviewed to establish if basic investigations had been performed and if so, their results.

Results Demographics: 19 F:16 M. Mean age 53, range 13-85. $83 \%$ of patients were referred by either general practitioners, general physicians or ENT surgeons. Features at presentation (\%): ENT (94), arthralgia (66), cutaneous (51), renal (40), ocular (29), pulmonary (20) and neurological (17). The results are presented in the table. In $29 \%(10 / 35)$ of patients at least one test was not performed. 11\% (4/35) of patients had hypertension (diastolic BP > 99) and in none were recommendations made for BP to be rechecked or treatment to be commenced. $6 \%(2 /$ 35 ) of patients with microscopic haematuria did not have urine microscopy or a renal biopsy performed (one patient also had an elevated creatinine). 9 patients had an abnormal CXR: 1 had evidence of previous tuberculosis, 4 had active pulmonary disease and 4 had further investigation to exclude infection.

\begin{tabular}{llllll}
\multicolumn{7}{l}{ Abstract FRI0163 Table 1} \\
\hline & BP & Urine & Creatinine & CXR & Biopsy \\
\hline Test performed (no. of pts) & $33 / 35$ & $33 / 35$ & $31 / 35$ & $31 / 35$ & $30 / 35$ \\
Test performed (\% of pts) & 94 & 94 & 89 & 89 & 86 \\
Test abnormal (\% of pts) & 11 & 46 & 23 & 26 & 87 \\
\hline Initial assessment of Wegener's granulomatosis (pts = patients).
\end{tabular}

Conclusion $71 \%$ of patients referred to a tertiary rheumatology centre and later confirmed to have WG received adequate basic investigation at presentation. Patients found to be hypertensive should have their BP repeated and those with haematuria should have urine microscopy performed. These results indicate that we should be more vigilant in performing basic tests in patients with suspected WG and in this regard our data have been presented to our department by means of an audio visual presentation and written information. The audit will be repeated in 2005 to reassess the recording of basic investigations in patients with WG.

\section{FRI0164 ANTIBODIES TO PROTEINASE-3, MYELOPEROXIDASE AND B2GPI}

${ }^{1} \mathrm{AA}$ Baranov, ${ }^{1} \mathrm{SY}$ Kirdyanov, ${ }^{2} \mathrm{EL}$ Nassonov, ${ }^{2} \mathrm{TV}$ Beketova, ${ }^{1} \mathrm{MS}$ Gurieva, ${ }^{1}$ OV Bashina, ${ }^{1}$ NE Abaytova. Internal Medicine, Medical Academy, Yaroslavl; ${ }^{2}$ Internal Medicine, Medical Academy, Moscow, Russia

\subsection{6/annrheumdis-2001.224}

\section{Background}

Objectives To investigate the relationship between antibodies to proteinase-3 (anti-PR3), myeloperoxidase (anti-MPO) and b2GPI (anti-b2GPI) in patients (pts) with systemic vasculitis and primary antiphospholipid syndrome (PAPS).

Methods The sera from 18 pts with polyarteritis nodosa (PAN), 18 pts with Takayasu's arteritis (TA), 21 pts with thromboangiitis obliterans (TAO), 21 pts with Henoch-Schonlein purpura (HSP), 